\title{
Top quark production at the LHC
}

\author{
Sinéad Walsh ${ }^{* \dagger}$ \\ Ghent University, Ghent, Belgium \\ E-mail: sinead.walsh@cern.ch
}

Studies of top quark production by the CMS and ATLAS collaborations are presented. The measurements have been performed with data collected at the Large Hadron Collider at a center-ofmass energy of $\sqrt{s}=7 \mathrm{TeV}$. The inclusive $t \bar{t}$ cross section is measured in the fully hadronic, dilepton and lepton+jets final states. A differential measurement of the $t \bar{t}$ cross section is also described. The cross sections for the t-channel and tW single top production processes are measured, and an upper limit is placed on the s-channel cross section. Standard Model predictions are found to be in agreement with all measurements. Recent results on searches for new physics related to top quark production are summarised.

The XIth International Conference on Heavy Quarks and Leptons,

June 11-15, 2012

Prague, Czech Republic

\footnotetext{
* Speaker.

$\dagger$ on behalf of the CMS and ATLAS Collaborations
} 


\section{Introduction}

In 2011 the LHC operated with a center-of-mass energy of $\sqrt{s}=7 \mathrm{TeV}$, delivering over $5 \mathrm{fb}^{-1}$ of data to both the CMS [1] and ATLAS [2] experiments. This allowed the experiments to probe previously unstudied properties of the top quark and top quark production.

The measurement of top quark production is important to verify the Standard Model. Measurements of the top quark pair production cross section test perturbative QCD predictions while single top production measurements probe electroweak interactions. The measurement of single top production cross sections allows for a direct determination of the CKM matrix element $\left|V_{t b}\right|$. Top quark production is interesting for new physics searches as new massive particles decaying to top quarks can be revealed in top production studies. Top production is also a significant background in many new physics searches, therefore a precise understanding of the production rate is required to constrain the top contribution.

In the following text an overview is provided of the top quark production measurements which have been performed by the CMS and ATLAS collaborations using data collected at the LHC in 2011. The Standard Model predictions are found to be in agreement with the measurements. Several results from new physics searches are also included.

\section{Simulation}

In CMS, $t \bar{t}$ events are simulated using MADGRAPH with matrix elements corresponding to up to three additional partons. The production of single top quarks is simulated with POWHEG. Generated events are processed with PYTHIA for parton showering. In ATLAS, the MC@NLO generator is used to simulate $t \bar{t}$ events. Simulated events are hadronised using the HERWIG shower model supplemented by the JIMMY underlying event model. The single top processes are simulated using the AcerMC program, with PYTHIA used for hadronisation. Both experiments use TAUOLA for the simulation of $\tau$ decays and assume a top quark mass of $172.5 \mathrm{GeV} / \mathrm{c}^{2}$.

\section{Object selection}

In both experiments, electron and muon candidates are required to be isolated, central objects with large transverse momentum, $\mathrm{p}_{\mathrm{T}}>20 \mathrm{GeV}$. Missing transverse energy, $\not_{T}$, is derived from the negative vectorial sum of the four momenta of objects in the event.

Jets are required to have high $\mathrm{p}_{\mathrm{T}}>20 \mathrm{GeV}$ and are reconstructed with the anti- $k_{T}$ clustering algorithm, with a radius parameter $\mathrm{R}=0.5$ in CMS and $\mathrm{R}=0.4$ in ATLAS. The identification of jets originating from $b$-quarks is performed using likelihood based algorithms.

In CMS, hadronic $\tau$ decays are reconstructed with the Hadron Plus Strips algorithm [3]. In ATLAS, hadronic $\tau$ decays are identified using the output of a boosted decision tree discriminant.

\section{Top pair production cross section}

In high energy collisions, top quarks are produced predominantly in pairs via the strong interaction. At a center-of-mass energy of $\sqrt{s}=7 \mathrm{TeV}, 85 \%$ of $t \bar{t}$ events are produced via gluon fusion 
and $15 \%$ are produced via $q \bar{q}$ annihilation. The approximate NNLO $t \bar{t}$ production cross section has been calculated as $163_{-10}^{+11} \mathrm{pb}$ [4] for proton-proton collisions at $\sqrt{s}=7 \mathrm{TeV}$.

Due to the short top quark lifetime, top quarks decay before they can be detected. Therefore, top quark events are examined through the identification of the top quark decay products. Since the top quark decays almost exclusively to a $\mathrm{W}$ boson and a $b$ quark, the final decay modes are determined by the $\mathrm{W}$ boson decay. There are three classes of $t \bar{t}$ final states. The fully hadronic final state, $t \bar{t} \rightarrow q q b q q b$, the dilepton final state, $t \bar{t} \rightarrow \ell v b \ell v b$, and the lepton+jets final state, $t \bar{t} \rightarrow q q b \ell v b$, where $q$ is a quark $(u, d, s, c, b), \ell$ is a lepton and $v$ is a neutrino.

\subsection{Fully hadronic channel $(t \bar{t} \rightarrow q q b q q b)$}

The challenge for studies of $t \bar{t}$ events in the fully hadronic final state is the separation of signal from QCD multijet background events. In both experiments, events are required to have at least six jets, at least two of which are identified as $b$-jets.

The cross section is extracted with a unbinned likelihood fit to the reconstructed top quark mass, $m_{t}$. The top quark mass distribution is chosen to separate the $t \bar{t}$ and multijet contributions and is reconstructed from a kinematic fit.

The signal $m_{t}$ distribution is provided by simulation while the distribution for multijet is derived from events where none of the selected jets are b-tagged. In CMS, since $b$-tagging can affect the $m_{t}$ shape, events with no $b$-tagged jets are weighted to reproduce the kinematics of the b-tagged jet events. In ATLAS, the effect of $b$-tagging on $m_{t}$ shape is studied using simulation to estimate the uncertainty on the background shape.

The measured $t \bar{t}$ cross section is $136 \pm 20$ (stat.) \pm 40 (syst.) \pm 8 (lumi.)pb in CMS [5] using $1.09 \mathrm{fb}^{-1}$ of data and $168 \pm 12$ (stat.) ${ }_{-57}^{+60}$ (syst.) \pm 6 (lumi.)pb in ATLAS [6] using $4.7 \mathrm{fb}^{-1}$ of data.

In both analyses the jet energy scale and $b$-tagging uncertainties are dominant. In the CMS analysis background modelling is a significant contributor while the ATLAS analysis is also affected by the uncertainty on the initial and final state radiation.

\subsection{Dilepton channel, with electrons and muons $(t \bar{t} \rightarrow \ell v b \ell v b, \ell=e, \mu)$}

The dilepton final state has the lowest production rate, however a distinct event signature allows for precise studies of the production rate. In both experiments, events are required to have large $Z_{T}$ and at least two jets. Di-electron and di-muon events where the invariant mass of the two leptons is small, or in the region of the $\mathrm{Z}$ boson mass, are removed.

In CMS, events are required to have at least one pair of oppositely signed leptons, where the lepton is either an electron or a muon, and at least one of the selected jets must be $b$-tagged. The cross section is extracted with a counting method by subtracting the expected number of background events from the number of observed data events.

In ATLAS, track-lepton candidates are defined by a high $\mathrm{p}_{\mathrm{T}}$ track with a series of quality cuts applied. Selected events must either have exactly one pair of oppositely charged leptons, or one lepton and one track-lepton candidate with opposite charge, referred to as a lepton+track event. The cross section is obtained with a profile likelihood technique combining seven channels, the dilepton and lepton+track channels where no jet is $b$-tagged, and the di-electron and di-muon channels with at least one $b$-tagged jet. 
Both analyses use a similar data driven technique to estimate the contribution from the dominant Drell-Yan background. A scale factor is derived from the ratio of data events to the expected number of DY events within the vetoed dilepton invariant mass window. This scale factor is used to correct the expected number of DY events in the signal region.

The $t \bar{t}$ cross section is measured as $169.9 \pm 3.9$ (stat.) \pm 16.3 (syst.) \pm 7.6 (lumi.)pb in the CMS analysis [7] with $1.14 \mathrm{fb}^{-1}$ of data and $176 \pm 5$ (stat. $)_{-11}^{+14}$ (syst.) \pm 8 (lumi.)pb in the ATLAS analysis [8] with $0.7 \mathrm{fb}^{-1}$ of data.

In the CMS analysis, $b$-tagging and pile-up are the most significant uncertainties. The uncertainty on the ATLAS result is dominated by the generator and jet energy scale/ $\mathbb{E}_{T}$ uncertainties.

\subsection{Dilepton channel, with a hadronically decaying tau $(t \bar{t} \rightarrow \tau \nu b \ell v b, \ell=e, \mu)$}

The $\tau$ final state is considered separately from the $e$ and $\mu$ dilepton final states as the $\tau$ decays, either leptonically or hadronically, before it can be detected. The following measurements consider the final state where the $\tau$ decays hadronically.

In both experiments, selected events are required to identify exactly one electron or muon, one $\tau$, large $E_{T}$ and at least two jets, with one or more $b$-tagged jet.

In CMS, the lepton and $\tau$ are required to have opposite sign charge. The cross section is extracted with a counting method by subtracting the expected number of background events from the number of observed data events.

In ATLAS, the contribution from events with same sign (SS) lepton and $\tau$ is subtracted from the opposite sign (OS) contribution, as background process are expected to have a similar amount of events in both regions. The cross section is extracted with a $\chi^{2}$ fit to an OS-SS boosted decision tree output distribution for events where the $\tau$ candidate has exactly one track and events where the $\tau$ candidate has at least two tracks. The region where the $\tau$ has more than one track is expected to be dominated by background, which helps to control the background in the signal region.

In CMS, the main background consists of events with at least three jets where one jet is misidentified as a $\tau$. In order to estimate this background from data, the probability that a jet is misidentified as a $\tau$ jet is determined. This probability is then applied to every jet in a preselected sample which requires at least three jets and has no $\tau$ requirement.

In ATLAS, after OS-SS subtraction the predominant remaining jet types are light-quark jets. A background boosted decision tree distribution is constructed from a data sample with zero $b$-tagged jets by subtracting the expected amount of true $\tau$ signal.

The CMS analysis [9] measures a $t \bar{t}$ cross section of $143 \pm 14$ (stat.) \pm 22 (syst.) \pm 3 (lumi.)pb with $2.2 \mathrm{fb}^{-1}$ of data, while the ATLAS analysis [10] measures a $t \bar{t}$ cross section of $186 \pm 13$ (stat.) \pm 20 (syst.) \pm 7 (lumi.)pb using $2.05 \mathrm{fb}^{-1}$ of data.

In CMS, the dominant systematic uncertainty is due to the $\tau$ identification and misidentification uncertainties. In ATLAS, the most significant sources of systematic uncertainty are the $b$-tagging uncertainty and the uncertainty on the initial and final state radiation.

\subsection{Lepton+jets channel, with an electron or muon $(t \bar{t} \rightarrow q q b \ell v b, \ell=e, \mu)$}

The balance of high production rate and clean event signature makes the lepton+jets final state ideal for precision measurements. In both experiments, considered events are required to have exactly one electron or muon and $\not_{T}$. 
In CMS, the secondary vertex mass distribution, $m_{\mathrm{SV}}$, is used to discriminate light jet events from heavy flavour jet events. The cross section is extracted with a simultaneous binned likelihood fit to the $m_{\mathrm{SV}}$ distribution for different jet multiplicities $(1,2,3,4, \geq 5)$ and number of $b$-tagged jets $(1, \geq 2)$. In ATLAS, the cross section is extracted from a simultaneous likelihood fit to a likelihood discriminant distribution for events with three, four and at least five jets. In both experiments, the low jet multiplicity regions, which are dominated by background, are used to control the background in the signal region.

In CMS, the normalisation of the multijet background is determined using a likelihood fit to the $Z_{T}$ distribution in data. In the muon channel, the $m_{\mathrm{SV}}$ shape for multijet background is derived from data by applying an inverse lepton isolation requirement. In ATLAS, the event yield for the dominant $\mathrm{W}+\mathrm{jets}$ background is obtained with a data-driven method which exploits the charge asymmetry of $\mathrm{W}$ boson production. The multijet background contribution is estimated using the matrix method.

In CMS [11] the measured $t \bar{t}$ cross section is $164.4 \pm 2.8$ (stat.) \pm 11.9 (syst.) \pm 7.4 (lumi.)pb with $1.09 \mathrm{fb}^{-1}$ of data. The ATLAS [12] analysis measures a $t \bar{t}$ cross section of $179 \pm 9.8($ stat + syst $) \pm$ 6.6(lumi.)pb with $0.70 \mathrm{fb}^{-1}$ of data.

In CMS, the uncertainties on the parton density function as well as the lepton identification uncertainties are the most significant systematic uncertainties. In ATLAS, the muon identification and generator uncertainties dominate the overall systematic uncertainty.

\subsection{Lepton+jets channel, with a hadronically decaying tau $(t \bar{t} \rightarrow q q b \tau \nu b)$}

The $t \bar{t}$ production cross section is measured in the lepton+jets $\tau$ final state where the $\tau$ decays hadronically. In CMS, selected events are required to have one identified $\tau, \not_{T}$ and at least four jets, at least one of which is $b$-tagged. The cross section is extracted with a likelihood fit to a neural network output distribution.

In ATLAS, the $\tau$ is not explicitly identified. Instead selected events are required to have at least five jets, at least two of which are $b$-tagged, and $E_{T}$. One of the selected jets is chosen as the $\tau$ candidate. A distribution of the number of charged tracks associated to the $\tau$ candidate is then used to separate true and misidentified $\tau$ candidates, as a $\tau$ decays preferentially to one or three charged particles. A likelihood fit to this distribution is used to extract the cross section.

In CMS, the multijet background is inferred from data by training the neural network in the region with zero $b$-tagged jets. In ATLAS, the $t \bar{t}$ template is derived from a control region where the $\tau$ candidate is replaced by a muon candidate and four jets are required. The multijet template is derived from a sideband region where the $E_{T}$ requirement is lowered such that it is orthogonal to the signal selection.

In CMS [13] a $t \bar{t}$ cross section of $156 \pm 12$ (stat.) \pm 33 (syst.) \pm 3 (lumi.)pb is measured using $3.9 \mathrm{fb}^{-1}$ of data, while ATLAS [14] measures $200 \pm 19$ (stat.) \pm 43 (syst.)pb using $1.67 \mathrm{fb}^{-1}$ of data.

In CMS, the systematic uncertainty is dominated by the jet energy scale and $\tau$ identification uncertainties. In ATLAS, initial and final state radiation and $b$-tagging are the most significant sources of systematic uncertainty.

\subsection{Combined results for top pair production}

In order to obtain a more precise estimate of the top quark pair production cross section, the 
experiments combine the results of the measurements performed in each channel with a likelihood fit. In both experiments, the fully hadronic, lepton+jets ( $e+$ jets, $\mu+$ jets $)$ and dilepton $(e e, \mu \mu, e \mu)$ results are combined. In CMS, the result in the dilepton $\mu \tau$ channel is also included.

The CMS combination [15] uses 0.8 to $1.1 \mathrm{fb}^{-1}$ of data and results in a $t \bar{t}$ cross section of

$$
165.8 \pm 2.2 \text { (stat.) } \pm 10.6 \text { (syst.) } \pm 7.8 \text { (lumi.) pb. }
$$

The ATLAS combination [16] is performed with 0.7 to $1.02 \mathrm{fb}^{-1}$ of data and results in a $t \bar{t}$ cross section of

$$
177 \pm 3 \text { (stat. })_{-7}^{+8}(\text { syst. }) \pm 7(\text { lumi. }) \text { pb. }
$$

The combined results, as well as the measurements in the individual channels, are compared to the Standard Model prediction in Figure 1. The Standard Model is found to be in agreement with all measurements.
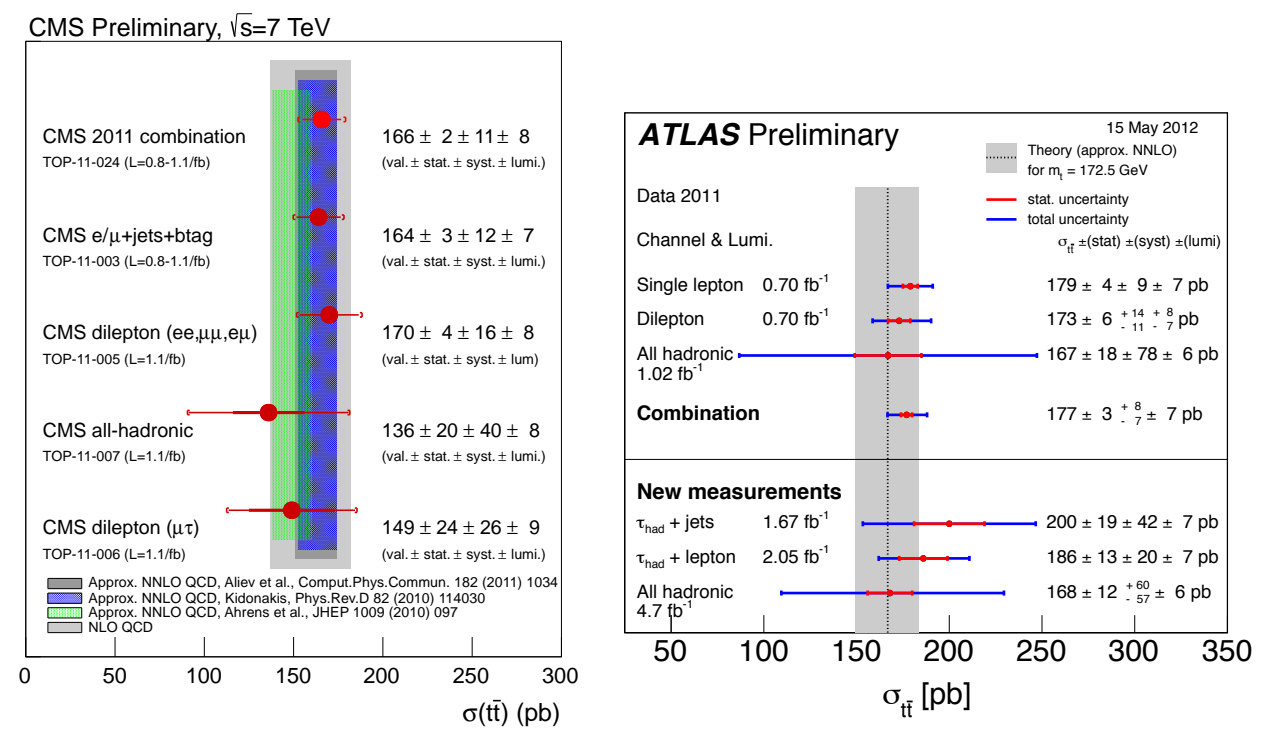

Figure 1: Results for measurements of the top quark pair production cross section performed in CMS (left) and ATLAS (right) compared with Standard Model predictions.

\subsection{Top pair differential cross section}

A measurement of the normalised differential top pair production cross section is performed by CMS [17] with $1.14 \mathrm{fb}^{-1}$ of data. A differential measurement is useful to verify top production mechanisms at $\sqrt{s}=7 \mathrm{TeV}$ within the scope of the Standard Model. Also, beyond the Standard Model physics could be uncovered in such measurements.

The cross section is measured in the lepton+jets and dilepton final states where the lepton is an electron or muon. In each channel the cross section is measured as a function of the $\mathrm{p}_{\mathrm{T}}$, (pseudo-)rapidity and invariant mass of the final state leptons, top quarks and the $t \bar{t}$ system. The measured differential cross section distributions are normalised to unity to cancel out all systematic 
effects affecting absolute normalisation of the cross section measurement. For each channel, for each observable $\mathrm{X}$, the cross section in each bin $\mathrm{i}$ is determined with Equation 4.1.

$$
\frac{1}{\sigma} \frac{d \sigma^{i}}{d X}=\frac{1}{\sigma} \frac{N_{\mathrm{Data}}^{i}-N_{\mathrm{BG}}^{i}}{\Delta_{X}^{i} \varepsilon^{i} \mathscr{L}}
$$

The kinematic properties of the top quark pair are determined with kinematic reconstruction algorithms. Bin-by-bin unfolding is used to correct effects from trigger and detection efficiencies and resolutions leading to migration of events across bin boundaries.

In the lepton+jets channel $Q^{2}$ and matching scale uncertainties, as well as the uncertainty on $b$-tagging, are the predominant sources of systematic uncertainty. In the dilepton channel the uncertainties on the background contributions and the lepton identification dominate. The Standard Model is found to be in agreement with all measurements.

\section{Single top production cross section}

Top quarks are produced individually via the electroweak interaction. There are three single top production mechanisms: the t-channel exchange of a $\mathrm{W}$ boson, the associated production of an on-shell $\mathrm{W}$ boson and a top quark, and the s-channel production and decay of a virtual $\mathrm{W}$ boson. The expected cross sections are calculated at NLO with NNLL corrections as $\sigma_{\mathrm{t}-\text { channel }}=64.6_{-2.0}^{+2.7}[18], \sigma_{\mathrm{tW}}=15.7 \pm 1.1[19]$ and $\sigma_{\mathrm{s}-\text { channel }}=4.6 \pm 0.2[20]$.

Studies of single top quark production are challenging due to the small production cross section and the difficulty in distinguishing signal from the $t \bar{t}$ event topology. However, measurements of single top production are a useful cross check of the Standard Model and allow for a direct measurement of the CKM matrix element $\left|V_{t b}\right|$. These measurements are also sensitive to many new physics models.

\section{1 t-channel $(t \rightarrow b \ell v, \ell=e, \mu)$}

In both experiments, t-channel events are selected by requiring exactly one electron or muon and exactly one $b$-tagged jet. The exclusion of events with more than one $b$-tagged jet reduces the $t \bar{t}$ background. The t-channel final state contains an additional light jet from the hard scattering process, often in the forward direction. Therefore, signal events are required to have an additional jet which is not $b$-tagged.

In CMS, a large reconstructed transverse $\mathrm{W}$ boson mass, $m_{T}(W)$, is required to select events in the muon channel while $\mathbb{E}_{T}$ is required in the electron channel. Signal events are also required to have a reconstructed top mass close to the expected top quark mass. The cross section is extracted from a likelihood fit to the pseudorapidity of the light jet, exploiting the forward tendency of the light jet to separate signal from background.

In ATLAS, $\not_{T}$ is required to select events. The cross section is extracted with a likelihood fit to a neural network output distribution in events with two and three jets. The three jet data sample is dominated by background, constraining the background in the two jet signal region.

In CMS, the multijet normalisation is derived with a likelihood fit to $m_{T}(W)$ in the muon channel and $\mathbb{E}_{T}$ in the electron channel. The multijet distribution is obtained with an inverted requirement on the muon isolation and the electron quality. The $\mathrm{W}+$ jets yield and distribution is 
extracted from the sideband region where the reconstructed mass is far from the expected top quark mass. In ATLAS, the multijet normalisation is derived with a likelihood fit to $\mathbb{E}_{T}$. The multijet distribution is obtained by replacing the lepton requirement with a jet requirement. The $\mathrm{W}+\mathrm{jets}$ normalisation is allowed to float freely in the fit to extract the t-channel cross section.

The CMS analysis [21] measures a t-channel cross section of 70.2 \pm 5.2 (stat.) \pm 10.4 (syst.) \pm 3.4(lumi.)pb with 1.14 to $1.51 \mathrm{fb}^{-1}$ of data. The ATLAS analysis [22] measures a t-channel cross section of $83 \pm 4$ (stat. $)_{-19}^{+20}$ (syst.)pb with $1.04 \mathrm{fb}^{-1}$ of data.

The value of the CKM element $\left|V_{t b}\right|$ is determined from the cross section measurements as $1.04 \pm 0.09$ (exp.) \pm 0.02 (th.) in CMS and $1.13_{-0.13}^{+0.14}$ in ATLAS. In CMS, the most significant sources of systematic uncertainty are jet energy scale and the W+jets heavy flavour extraction. In ATLAS, initial and final state radiation and $b$-tagging uncertainties dominate.

\section{2 tW channel $(t W \rightarrow \ell v b \ell v, \ell=e, \mu)$}

The rate of top quark production at the LHC in 2011 allowed for the first observation of the tW process. The event selection is similar to the $t \bar{t}$ dilepton selection detailed in Section 4.2, with the exception of the jet requirement. Events with at least one jet are considered.

In CMS, the region with exactly one $b$-tagged jet is considered as the signal region. Two regions are defined to control the $t \bar{t}$ background in the cross section extraction: one with exactly two $b$-tagged jets and one with exactly two jets, one of which is $b$-tagged. The cross section is extracted with a simultaneous likelihood fit in the signal region and the two background regions. In ATLAS, the cross section is extracted with a likelihood fit to a boosted decision tree output in the one, two and at least three jet samples, where the two and three jet samples are used to control the background in the signal region.

In both experiments, the Drell-Yan contribution is estimated using a data driven method similar to the one described for the $t \bar{t}$ dilepton analyses in Section 4.2.

The CMS analysis [23] observes an excess of events over the expected background with a significance of $2.7 \sigma$, using $2.1 \mathrm{fb}^{-1}$ of data. The measured value of the cross section and $68 \% \mathrm{CL}$ interval is $22_{-7}^{+9}$ (stat. + syst.)pb. The result of ATLAS analysis [24], performed with $2.05 \mathrm{fb}^{-1}$ of data, is incompatible with a background only hypothesis at the $3.3 \sigma$ level. The measured cross section is $16.8 \pm 2.9$ (stat.) \pm 4.9 (syst.)pb and the CKM matrix element $\left|V_{t b}\right|$ is determined as $1.03_{-0.19}^{+0.16}$.

In CMS, the most significant sources of systematic uncertainty are the $b$-tagging uncertainties and the difference between the DR and DS scheme used to define tW signal in simulation. In ATLAS, the jet energy scale and parton shower uncertainties dominate the systematic uncertainty.

\section{3 s-channel $(t b \rightarrow \ell v b b, \ell=e, \mu)$}

A search for the s-channel single top production mode, which has not previously been observed by itself, is performed by ATLAS [25] with $0.7 \mathrm{fb}^{-1}$ of data. Selected events are required to have one lepton, $Z_{T}$ and two $b$-tagged jets.

Extraction of the s-channel single top quark signal is challenging as it is overwhelmed by background processes with the same final states, such as $\mathrm{W}+\mathrm{jets}$ and $t \bar{t}$. A simple cut based method is used to search for the s-channel signal. After the final selection 296 data events are observed compared with a prediction of 285 events, with a predicted signal purity of $6 \%$. The s-channel production cross section is determined with a counting experiment modelled by a likelihood function. 
The multijet normalisation is determined with a likelihood fit to the $E_{T}$ distribution in data. The multijet template shape is obtained with an inverted lepton isolation requirement. The $\mathrm{W}+\mathrm{jets}$ normalisation is taken as the difference between the number of data events and the number of events estimated for non $\mathrm{W}+$ jets backgrounds in each jet multiplicity bin. The $\mathrm{W}+$ jets flavour composition is estimated from data samples with small signal contamination: a one $b$-tagged jet sample, a two non $b$-tagged jets sample and a sample with two jets, one of which is $b$-tagged.

An upper limit of $\sigma_{s}<26.5 \mathrm{pb}$ is set on the observed s-channel cross section at 95\% CL. The expected upper limit is $\sigma_{s}<20.5 \mathrm{pb}$.

The most significant sources of systematic uncertainty are the generator and $b$-tagging uncertainties as well as the uncertainty on the multijet normalisation.

\section{New Physics Searches}

Extensive searches for new physics have been performed at the LHC at $\sqrt{s}=7 \mathrm{TeV}$. These searches have resulted in many lower limits being placed on the masses of new particles, as well as upper limits on the cross section of new physics processes. Several results which involve the top quark in new physics processes are shown in Table 1.

\begin{tabular}{llr|lr}
\hline Search & CMS & 95\% CL excluded & ATLAS & 95\% CL excluded \\
\hline$t b$ resonances & & & {$[29]$} & $m_{W_{R}^{\prime}}<1.13 \mathrm{TeV}$ \\
$t \bar{t}$ resonances & {$[26]$} & $m_{Z^{\prime}}<1.7 \mathrm{TeV}$ & {$[30]$} & $m_{Z^{\prime}}<0.88 \mathrm{TeV}$ \\
& & KK-gluon $<1.4 \mathrm{TeV}$ & & KK-gluon $<1.1 \mathrm{TeV}$ \\
$b^{\prime} b^{\prime} \rightarrow W t W t$ & & & {$[31]$} & $m_{b^{\prime}}<480 \mathrm{GeV}$ \\
same sign top & {$[27]$} & $\sigma>0.61 \mathrm{pb}$ & {$[32]$} & $\sigma>1.4-2.0 \mathrm{pb}$ \\
$t^{\prime}$ & {$[28]$} & $m_{t^{\prime}}<490 \mathrm{GeV}$ & {$[33]$} & $m_{t^{\prime}}<404 \mathrm{GeV}$ \\
\hline Further results & {$[34],[35],[36]$} & {$[38]$} & \\
\hline
\end{tabular}

Table 1: Results from new physics searches performed in CMS and ATLAS with data collected at the LHC at $\sqrt{s}=7 \mathrm{TeV}$.

\section{Conclusions}

A comprehensive examination of the top quark production processes has been performed by the CMS and ATLAS collaborations with data collected at the LHC at $\sqrt{s}=7 \mathrm{TeV}$. Precision measurements of $t \bar{t}$ production have been obtained in the dilepton and lepton+jets final states. First measurements of $t \bar{t}$ fully hadronic, $\tau$ final state and differential cross sections as well as single top cross sections have been performed. All measurements are found to be in agreement with Standard Model predictions. Searches performed by the CMS and ATLAS experiments have resulted in many limits on new physics processes involving the top quark at $\sqrt{s}=7 \mathrm{TeV}$.

\section{References}

[1] CMS Collaboration, "CMS experiment at the CERN LHC", JINST 3 (2008) S08004. 
[2] ATLAS Collaboration, "ATLAS experiment at the CERN LHC", JINST 3 (2008) S08003.

[3] CMS Collaboration, JINST 7 (2012) P01001.

[4] N. Kidonakis, Phys. Rev. D 82 (2010) 114030, arXiv:1009.4935

[5] CMS Collaboration, CMS-PAS-TOP-11-007, http://cdsweb.cern.ch/record/1371755

[6] ATLAS Collaboration, ATLAS-CONF-2012-031, http://cdsweb.cern.ch/record/1432196

[7] CMS Collaboration, CMS-PAS-TOP-11-005, http://cdsweb.cern.ch/record/1377323

[8] ATLAS Collaboration, JHEP 1205 (2012) 059, arXiv: 1202.4892

[9] CMS Collaboration, CERN-PH-EP-2012-078, http://cdsweb.cern.ch/record/1436060

[10] ATLAS Collaboration, CERN-PH-EP-2012-102, arXiv:1205.2067

[11] CMS Collaboration, CMS-PAS-TOP-11-003, http://cdsweb.cern.ch/record/1386709

[12] ATLAS Collaboration, ATLAS-CONF-2011-121, http://cdsweb.cern.ch/record/1376413

[13] CMS Collaboration, CMS-PAS-TOP-11-004, http://cdsweb.cern.ch/record/1446652

[14] ATLAS Collaboration, ATLAS-CONF-2012-032, http://cdsweb.cern.ch/record/1432198

[15] CMS Collaboration, CMS-PAS-TOP-11-024, http://cdsweb.cern.ch/record/1401250

[16] ATLAS Collaboration, ATLAS-CONF-2012-024, http://cdsweb.cern.ch/record/1430733

[17] CMS Collaboration, CMS-PAS-TOP-11-013, http://cdsweb.cern.ch/record/1422425

[18] N. Kidonakis, Phys. Rev. D 83 (2011) 091503, arXiv:1103.2792

[19] N. Kidonakis, Phys. Rev. D 82 (2010) 054018, arXiv:1005.4451

[20] N. Kidonakis, Phys. Rev. D 81 (2010) 054028, arXiv:1001.5034

[21] CMS Collaboration, CMS-PAS-TOP-11-021, http://cdsweb.cern.ch/record/1430743

[22] ATLAS Collaboration, CERN-PH-EP-2012-082, arXiv:1205.3130

[23] CMS Collaboration, CMS-PAS-TOP-11-022, http://cdsweb.cern.ch/record/1385552

[24] ATLAS Collaboration, CERN-PH-EP-2012-117, arXiv:1205.5764

[25] ATLAS Collaboration, ATLAS-CONF-2011-118, http://cdsweb.cern.ch/record/1376410

[26] CMS Collaboration, CMS-PAS-TOP-11-009, http://cdsweb.cern.ch/record/1429634

[27] CMS Collaboration, CERN-PH-EP-2012-127, arXiv:1205.3933

[28] CMS Collaboration, CMS-PAS-EXO-11-054, http://cdsweb.cern.ch/record/1387505

[29] ATLAS Collaboration, CERN-PH-EP-2012-087, arXiv:1205.1016

[30] ATLAS Collaboration, CERN-PH-EP-2012-122, arXiv:1205.5371

[31] ATLAS Collaboration, CERN-PH-EP-2011-230, arXiv:1202.6540

[32] ATLAS Collaboration, JHEP 1204 (2012) 069, arXiv:1202.5520

[33] ATLAS Collaboration, Phys.Rev.Lett. 108 (2012) 261802, arXiv:1202.3076

[34] CMS Collaboration, CMS-PAS-EXO-11-050, http://cdsweb.cern.ch/record/1376672

[35] CMS Collaboration, CMS-PAS-EXO-11-051, http://cdsweb.cern.ch/record/1369546

[36] CMS Collaboration, CMS-PAS-EXO-11-006, http://cdsweb.cern.ch/record/1370237

[37] CMS Collaboration, CMS-PAS-EXO-11-055, http://cdsweb.cern.ch/record/1376673

[38] ATLAS Collaboration, CERN-PH-EP-2012-008, arXiv:1202.3389 